\title{
Scald-Induced Necrobiosis Lipoidica in a Patient with Diabetes Mellitus and Psoriasis
}

\author{
Hoshiko Ito Sadao Imamura \\ Department of Dermatology, Jyujyo Takeda Rehabilitation Hospital, Kyoto, Japan
}

\section{Key Words}

Necrobiosis lipoidica $\cdot$ Scald $\cdot$ Diabetes mellitus $\cdot$ Psoriasis $\cdot$ Koebner phenomenon

\begin{abstract}
The Koebner phenomenon (KP) was first introduced by Heinrich Koebner in the 1870s to describe the appearance of psoriatic lesions following trauma in psoriasis patients. KP has since been defined in numerous diseases, including necrobiosis lipoidica diabeticorum (NLD). Since most Koebnerized dermatological lesions can localise to a site of previous trauma, Weiss et al. (Eur Acad Dermatol Venereol 2002;16:241-248) classified them into four categories (I-IV) according to the Boyd-Nelder classification (Int J Dermatol 1990;29:401-410) system. In this system, necrobiosis lipoidica is classified as category III, which includes diseases that occasionally localise at the site of trauma. We report a case of NLD that developed after scald in a psoriasis patient. NLD after trauma has often been reported, but this is the first case of NLD that coincidentally occurred at a scald site in a psoriasis patient.
\end{abstract}

\section{(C) 2016 The Author(s)}

Published by S. Karger AG, Basel

\section{Case Report}

A 49-year-old man was referred to our dermatology department in 2014 with a discoloured plaque that had two small ulcers on the frontal aspect of his right lower leg in 2014 (fig. 1a). He was scalded on his right leg with hot water in 2011 (fig. 1b) and this discoloured lesion coincided with a previous scald, except for a small shallow burn that healed with slight depigmentation. The skin lesion showed central greyish atrophy, surrounded by a violaceous border that consisted of telangiectasia. The man had a medical history of type II diabetes mellitus (DM), hypertension, hyperlipidaemia, psoriasis and paralysis on the right

\section{KARGER}

Hoshiko Ito, MD

Department of Dermatology, Jyujyo Takeda Rehabilitation Hospital

32-Hattanda-cho kissyoin minami-ku

Kyoto 601-8325 (Japan)

E-Mail h-ito@takedahp.or.jp 
Ito and Imamura: Scald-Induced Necrobiosis Lipoidica in a Patient with Diabetes Mellitus and Psoriasis

side of his body due to transverse myelitis. Typical psoriatic plaques were scattered on the upper and lower extremities, trunk, and head. His DM had been well managed. A skin biopsy was performed from the atrophic area adjacent to the violaceous erythema and showed dense infiltration of lymphocytes, histiocytes, and neutrophils in the dermis (fig. 2a, b) as well as degenerated collagen fibres with signs of palisaded arrangements of the infiltrating cells (fig. 2c). Apparent granulomatous structures with giant cells were not observed. Gray et al. [1] reported a prominent palisaded arrangement of cells in 37\% of necrobiosis lipoidica diabeticorum (NLD) patients, whereas Muller and Winkelmann [2] observed palisading granuloma in $77 \%$ of the biopsy specimens from NLD patients. The divergent figures in these reports may reflect differences in the authors' interpretation of what constitutes significant palisading of cells [2]. Llajam [3] reported a case of NLD after a scald in a patient without psoriasis, in which no granulomatous structure was observed.

In our case, periodic acid-Schiff stain, Grocott's methenamine silver stain, and direct immunofluorescence studies showed negative results. Routine blood tests were within the normal range except for mild kidney dysfunction, though urinalysis was normal. The patient's chest radiograph was normal. On the basis of these clinical and histological features, we diagnosed NLD.

\section{Discussion}

NLD that develops after a trauma has often been reported. Our patient had psoriasis, which often indicates the Koebner phenomenon (KP). The fact that NLD, not psoriasis, arose from the site of the scald in this psoriasis patient was an interesting finding. NL in a patient with psoriasis is extremely rare and only two cases have been reported in the literature [4, 5]. Nagato et al. [4] reported a case of NL in a psoriasis patient, but NL did not localise to the trauma lesions. Abraham et al. [5] reported a patient who developed NLD, psoriasis, granuloma annulare, and vitiligo, but the appearance of NLD was not proved to be related with KP, since there was no record of previous trauma in the lesion. Our case is unique in that the NLD lesions closely coincided with the trauma site in a psoriasis patient.

$\mathrm{KP}$ is commonly associated with psoriasis, although it has been described in many other diseases. Boyd and Nelder [6] classified the three disease processes as true isomorphic response of Koebner (category I): these include psoriasis, lichen planus, and vitiligo. It is important to distinguish these from the pseudo-Koebner responses caused by infectious agents, such as molluscum contagiosum and verrucae vulgaris (category II). Weiss et al. [7] classified NL as category III, which includes diseases that occasionally localise to trauma lesions. Our case adds some insight into the mechanisms of KP. The shallow small scald ulcer did not develop NLD, but only depigmentation, while the deep ulcer induced NLD. The relationship between the type of trauma and KP has been previously investigated [8], and it appears that there must be epidermal injury in order to experimentally induce a Koebner reaction in a susceptible individual. Our case suggests that the depth of the ulcer influences the development of the NLD lesions. Even though DM was well managed in our patient, it may have had some influence in the mechanism of KP. Miller [8] suggested that positive KP reactivity correlated with psoriasis activity; however, in our case, the psoriasis was not very active with a few skin lesions (PASI: psoriasis area and severity index $<5$ ).

We treated the NLD lesions with alprostadil-alfadex ointment. The small ulcers healed after 1 week, followed by the slight decrease of peripheral telangiectasia about 1 month later. To date, no treatment has been proven effective for NLD in large studies; however, in our 
case, alprostadil-alfadex ointment slightly reduced the violaceous erythema. Further cases are needed to prove the effectiveness of this ointment.

\section{Acknowledgements}

We thank Dr. Natsuko Okamoto and Dr. Eiichi Okamoto for sharing the image.

\section{Statement of Ethics}

We have no ethical conflicts to disclose.

\section{Disclosure Statement}

We have no conflicts of interest in relation to the publication of this paper.

\section{References}

1 Gray HR, Graham JH, Johnson WC: Necrobiosis lipoidica: a histopathological and histochemical study. J Invest Dermatol 1965;44:369-380.

2 Muller SA, Winkelmann RK: Necrobiosis lipoidica diabeticorum histopathologic study of 98 cases. Arch Dermatol 1966;94:1-10.

-3 Llajam MA: Koebner's phenomenon and necrobiosis lipoidica diabeticorum. Br J Clin Pract 1990;44:765.

4 Nagato H, Demitsu T, Komatsu M, Tanita M, Manabe M, Yoneda K: A case of necrobiosis lipoidica in a patient with psoriasis (in Japanese). Rinsho Derma 2000;42:1991-1993.

5 Abraham Z, Lahat N, Kinarty A, Feuerman EJ: Psoriasis, necrobiosis lipoidica, granuloma annulare, vitiligo and skin infections in the same diabetic patient. J Dermatol 1990;17:440-447.

6 Boyd AS, Nelder KH: The isomorphic response of Koebner. Int J Dermatol 1990;29:401-410.

7 Weiss G, Shemer A, Trau H: The Koebner phenomenon: review of the literature. J Eur Acad Dermatol Venereol 2002;16:241-248.

-8 Miller RA: The Koebner phenomenon. Int J Dermatol 1982;21:192-197. 


\section{Case Reports in Dermatology}

Ito and Imamura: Scald-Induced Necrobiosis Lipoidica in a Patient with Diabetes Mellitus and Psoriasis
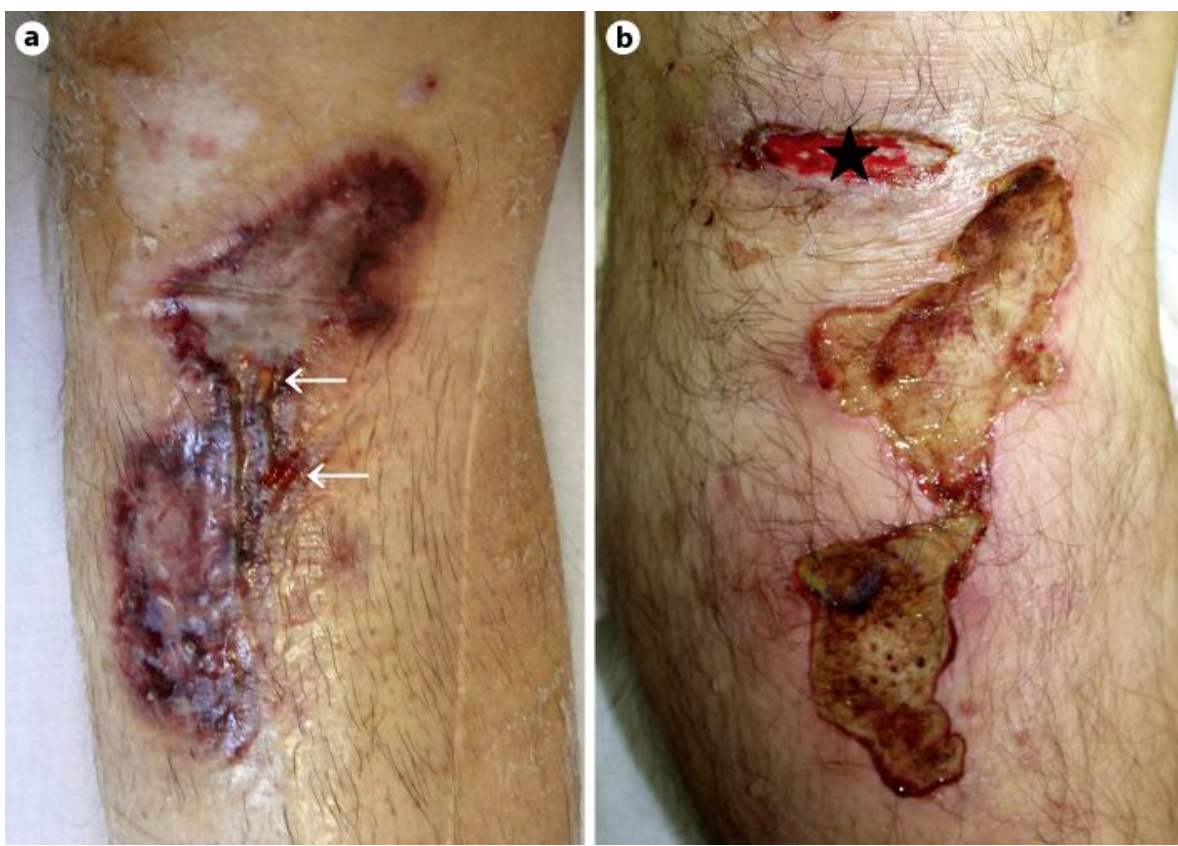

Fig. 1. a Clinical appearance of the lesion in 2014: an atrophic plaque with a violaceous border and two small ulcers (arrows). b Clinical appearance of the scald in 2011: a small ulcer indicated by a star ( $\star$ ) healed without developing NL but left a slight depigmentation.
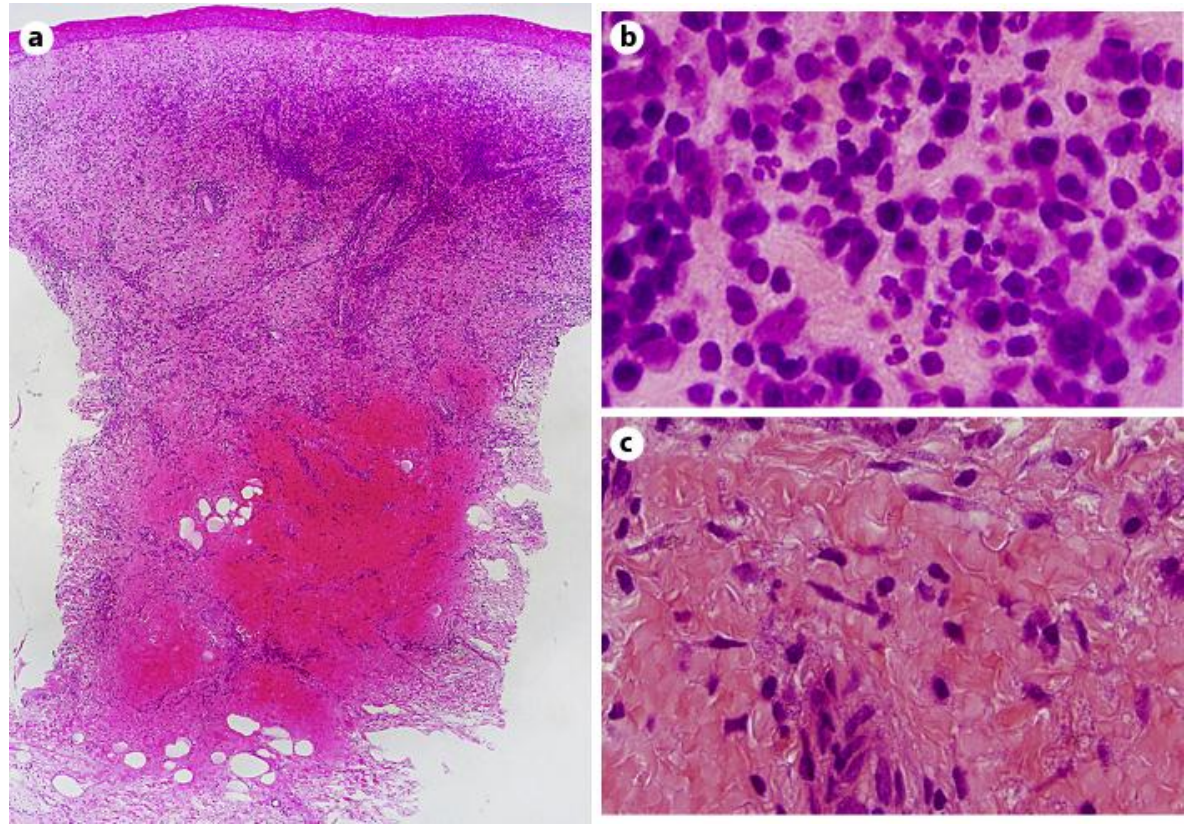

Fig. 2. a Low-power histological view showing a dense infiltration in the dermis and degeneration of the collagen fibres in the deep dermis [hematoxylin and eosin stain (HE), original magnification $\times 16$ ]. $\mathbf{b}$ Highpower histological view showing infiltration of lymphocytes, histiocytes and neutrophils in the upper dermis $(H E$, original magnification $\times 200)$. c High-power histological view showing the degenerated collagen fibres with palisading by mononuclear cells in the deep dermis (HE, original magnification $\times 200$ ). 